\title{
Isolated Recovery of Adductor Muscle Function Following Bilateral Recurrent Laryngeal Nerve Injuries
}

\author{
Ádám Bach, MD (1); Balázs Sztanó, MD, PhD ㅎ; Vera Matievics, MD; Zsófia Bere, MD, PhD; \\ Fabian Volk, MD; Andreas Müller, MD 웅 Gerhard Förster, MD; Paul F. Castellanos, MD, FCCP; \\ László Rovó, $\mathrm{MD}, \mathrm{PhD}$
}

\begin{abstract}
Objectives/Hypothesis: The aim of this study was to analyze the phoniatric and respiratory outcomes of a subset of bilateral vocal cord paralysis (BVCP) patients who were all treated with unilateral endoscopic arytenoid abduction lateropexy (EAAL). EAAL is a nondestructive, minimally invasive glottis widening operation, which does not damage either the surgically treated or the contralateral vocal cord. Therefore, it does not impair the regeneration potential of the recurrent laryngeal nerve.

Study Design: Case series.

Methods: Ten out of 21 BVCP patients who were treated with EAAL showed signs of isolated adduction recovery at 1 year and were chosen for this study. Functional results (objective and subjective voice analysis, spirometric measurement) and vocal cord movements were assessed preoperatively, 1 week and 1 year after EAAL. Laryngeal electromyography was performed on the 12th postoperative month.

Results: The volitional adductor movement seen on laryngoscopy was corroborated by laryngeal electromyography evaluation. Peak inspiratory flow increased significantly after EAAL. Quality-of-life scores also showed high patient satisfaction. Shimmer showed consistent improvement along with harmonic-to-noise ratio and average maximal phonation time in parallel with the improving vocal cord movement. Complex voice analysis and subjective self-evaluation tests also demonstrated significant improvement.

Conclusions: EAAL, as a minimally invasive, nondestructive airway widening technique, does not interfere with the potential regeneration process that can still occur after BVCP, allowing for laryngeal functional recovery. It is a safe and effective treatment for BVCP that allows a simple solution with good phonatory, swallowing, and respiratory benefits by unilateral passive and reversible vocal cord lateralization.
\end{abstract}

Key Words: Quality of life, dysphonia, airway stenosis, laryngeal electromyography, vocal cord paralysis.

Level of Evidence: 4.

Laryngoscope, 00:1-7, 2018

\section{INTRODUCTION}

The treatment of bilateral vocal cord immobility is still one of the biggest challenges in laryngology. This has been attributed the zero-sum balance between the need to improve breathing at the expense of sacrificed voice and swallowing functions. ${ }^{1}$ Although no debate exists about that the need for an immediate and stable airway is paramount, it is possible to achieve this while sustaining a satisfactory voice and protection from aspiration by using a minimally invasive, nondestructive, and reversible glottis-widening surgical technique. ${ }^{2}$ This way the zero-sum game can become a win-win.

From the Department of Otorhinolaryngology-Head and Neck Surgery (Á.B., B.S., V.M., Z.B., L.R.), University of Szeged, Szeged, Hungary; Department of Otorhinolaryngology (F.v.), Jena University Hospital, Jena, Germany; Department of Otorhinolaryngology (A.M., G.F.), SHR WaldKlinikum Gera, Gera, Germany; and the Northern Light Health Medical Center (P.F.C.), Bangor, Maine, U.S.A.

Editor's Note: This Manuscript was accepted for publication on November 5, 2018.

The authors have no funding, financial relationships, or conflicts of interest to disclose.

Send correspondence to Ádám Bach, MD, Tisza Lajos krt. 111., 6725, Szeged, Hungary. E-mail: bach.adam@med.u-szeged.hu

DOI: 10.1002/lary.27718
Theories have changed fundamentally since the Semon-Rosenbach and Wagner-Grossman hypotheses about the reason for the typical paramedian position of the vocal cord(s) after recurrent laryngeal nerve (RLN) injury. ${ }^{3-5}$ Animal studies clearly demonstrate that the RLN has a strong potential to regenerate after injury. It has also become evident that most patients with RLN injury attain varying levels of spontaneous recovery. ${ }^{6-10}$ Partial or even total recovery of vocal cord movement can be seen in untreated patients with unilateral or even bilateral vocal cord palsy (BVCP). Currently, the widely accepted conventional static glottis-widening techniques are based on the partial or complete resection of the arytenoid cartilage and/or the vocal cord. Clinical signs of recovery, which are commonly in the form of vocal cord adduction, are not easily detected in patients who are treated with glottis and/or arytenoid resection procedures, although laryngeal electromyography (LEMG) can demonstrate some degree of reinnervation., ${ }^{4,6}$ Thus, despite the neural regeneration, vocal cord movement may not be physically possible or may be undetectable and ineffective because of the surgery performed. 
By contrast, the nondestructive, minimally invasive, reversible glottis-widening technique, known as endoscopic arytenoid abduction lateropexy (EAAL), does not damage either the surgically treated or the contralateral vocal cord, and therefore can take advantage of the regeneration of the RLN. ${ }^{11-16}$ This way, the devastating clinical and physiological conundrum resulting from BVCP is reversed; instead of being closed, the glottis is surgically opened, but it is able to close during phonation or swallowing processes. A thorough analysis of LEMG, spirometric, and acoustic tests are required to assess the comparative advantage of this surgical method compared to conventional interventions performed for BVCP.

\section{MATERIALS AND METHODS}

\section{Patients}

Twenty-one consecutive patients were diagnosed and treated with unilateral EAAL because of BVCP caused by thyroid surgery. Ten of the $21(48 \%)$ patients (nine females and one male, mean age $=54.9$ years, range $=34-75$ years) showed endoscopic signs of only adduction recovery during the 1-year follow-up period; this homogenous group of patients with adduction-only recovery without any abduction recovery was selected for this report (Table I, Fig. 1). The other 11 patients of the original 21 had unilateral (four patients) or bilateral (two

\begin{tabular}{|c|c|c|c|}
\hline \multicolumn{4}{|c|}{$\begin{array}{l}\text { TABLE I. } \\
\text { Data of BVCP Patients With Adduction Recovery (1 Year } \\
\text { Postoperative). }\end{array}$} \\
\hline PatientNo./Age, yr/Sex & From BVCPto EAAL & VocalFold & Adduction \\
\hline \multirow[t]{2}{*}{$1 / 52 / F$} & 2 months & Right & 3 \\
\hline & & Left* & $1^{*}$ \\
\hline \multirow[t]{2}{*}{$2 / 46 / F$} & 1 day & Right* & $3^{*}$ \\
\hline & & Left & 1 \\
\hline \multirow[t]{2}{*}{$3 / 65 / F$} & 3 days & Right* & $1 *$ \\
\hline & & Left & 1 \\
\hline \multirow[t]{2}{*}{$4 / 39 / F$} & 3 months & Right & 3 \\
\hline & & Left* & $1^{*}$ \\
\hline \multirow[t]{2}{*}{$5 / 75 / \mathrm{M}$} & 4 months & Right & 3 \\
\hline & & Left* & $0 *$ \\
\hline \multirow[t]{2}{*}{ 6/35/F } & 1 month & Right & 3 \\
\hline & & Left* & $1^{*}$ \\
\hline \multirow[t]{2}{*}{ 7/68/F } & 2 days & Right & 3 \\
\hline & & Left* & $1 *$ \\
\hline \multirow[t]{2}{*}{$8 / 34 / F$} & 1 month & Right & 3 \\
\hline & & Left* & $2 *$ \\
\hline \multirow[t]{2}{*}{ 9/71/F } & 1 day & Right & 2 \\
\hline & & Left* & $3^{*}$ \\
\hline \multirow[t]{2}{*}{$10 / 64 / F$} & 2 months & Right & 3 \\
\hline & & Left* & 0* \\
\hline
\end{tabular}

Third column: grade of endoscopically detected adduction movement per vocal cord. 0: no adduction, 1: mild adduction, 2: complete adduction, 3 : adduction beyond the middle line of the glottis. Two of the 10 patients (patients 2 and 9) showed more adduction in the lateralized side than the unoperated side.

*The lateralized vocal cord.

$\mathrm{BVCP}=$ bilateral vocal cord paralysis; EAAL = endoscopic arytenoid abduction lateropexy; $\mathrm{F}=$ female; $\mathrm{M}$ = male.

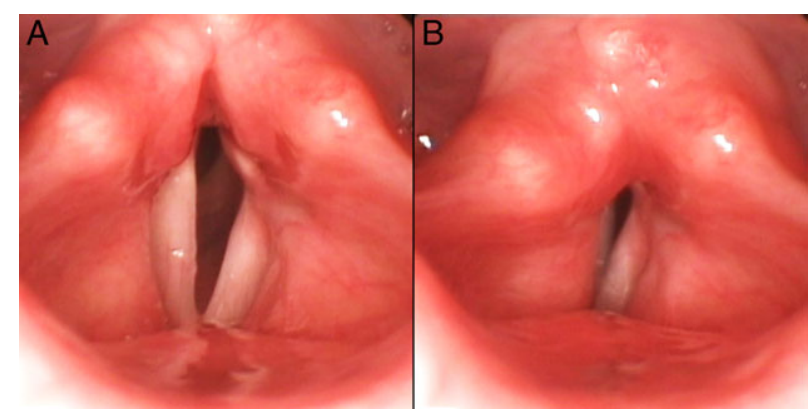

Fig. 1. Recovery of right vocal cord volitional adduction after bilateral vocal cord paralysis treated with left-sided endoscopic arytenoid abduction lateropexy (patient 6, postoperative 12th month, endoscopic view) with the ability to cross the midline. (A) Wide glottis during inspiration. (B) Cross-midline adduction of the right vocal cord. [Color figure can be viewed in the online issue, which is available at www.laryngoscope.com.] [Color figure can be viewed in the online issue, which is available at www.laryngoscope.com.]

patients) abduction recovery or failed to have any motion recovery (five patients) during the 1-year interval, indicating an entirely distinct clinical and physiological state as compared to the 10 with unilateral or bilateral adductor recovery.

In the 10 patients with isolated adductor recovery (with no abductor function detected), functional status was assessed preoperatively (as possible), 1 week, and 1 year after EAAL. LEMG of the vocal cords was performed on the 12th postoperative month to examine the neurophysiologic basis of the glottic movements. Preoperative respiratory and voice assessment was not feasible due to severe dyspnea or orotracheal intubation in four of the 10 patients. The study was approved by our institutional ethics committee (registration number 47/2015). Statistical analysis (analysis of variance) was performed with SigmaPlot 13 scientific graphing and statistical analysis software (Systat Software Inc., San Jose, CA).

\section{Voice Assessment}

Voice assessment was performed according to our previously published protocol and was based on the guidelines published by the Committee on Phoniatrics of the European Laryngological Society. ${ }^{17}$

Acoustic measurements. Voice samples were recorded within a sound-insulated room with a high-sensitivity condenserhead microphone (Audio-Technica ATM 73 ac; Audio-Technica Corp., Tokyo, Japan). The acoustic data were obtained by having three samples of a sustained /a/ at a comfortable pitch/loudness spontaneously chosen by each patient and lasting approximately 3 seconds. The averages derived from three samples of each phonation of each patient served as the basis for the analysis. Jitter $\%$, shimmer $\%$, fundamental frequency, harmonics-to-noise ratio (HNR), and maximum phonation times (MPT) were analyzed using Praat 5.3.37 software (www.praat.org).

Subjective self-evaluation. To assess the patients' voices related to their quality of life, the Hungarian version of the Voice Handicap Index (VHI) was used. ${ }^{18}$ The questionnaire contained 30 items in three subscales (10 items in each subscale: functional, emotional, and physical). Answers were given in a fivepoint scale ranging from 0 (never) to 4 (always). The overall VHI score (raw score) can be used to grade subjective handicap from no handicap (raw score $=0-14$ ), mild handicap (raw score $=15-28$ ), moderate handicap (raw score $=29-50$ ), and severe handicap $($ raw score $=51-120) .{ }^{19}$ 
Complex calculated linear index for evaluation of dysphonia. The Dysphonia Severity Index (DSI) was used in the study to measure the overall quality of the voicing. ${ }^{20}$ The DSI is designed to establish an objective and quantitative measurement of the perceived voice quality to assess the efficacy of therapy among dysphonic patients. The index is based on the weighted combination of objective acoustic and aerodynamic parameters, based on objective measures. DSI $=(0.13 \times$ MPT $)+(0.0053 \times$ highest frequency $)-(0.26 \times$ minimum intensity $)-(1.18 \times$ jitter $\%)+12.4$. A normal voice $\geq 5$ and a severely dysphonic voice scores $\leq-5$.

Friedrich Dysphonia Index. To evaluate the results, we also used the Friedrich Dysphonia Index (FDI). ${ }^{21}$ This index assesses hoarseness ( $\mathrm{G}$ value from GRBAS scale [grade, roughness, breathiness, asthenia, strain]), frequency range, dynamic range, maximum phonation time, and impairment of communication (from the VHI). For each category, a score ranging from 0 (normal) to 3 (severe) is assigned. The mean value of these scores is used to grade the dysphonia.

\section{Respiratory Assessment}

Peak inspiratory flow (PIF) is one of the characteristic and commonly used inspiratory parameters that describes the efficacy of glottis-enlarging procedures. ${ }^{22}$ Spirometric measurements were performed by using a Thor Spirotube-PC spirometer (THOR Laboratories Kft., Székesfehérvár, Hungary). The functional outcomes of the surgery in terms of breathing, voice,

\section{TABLE II.}

Results of Laryngeal Electromyography 1 Year After Recurrent Laryngeal Nerve Injury.

\begin{tabular}{|c|c|c|c|c|c|c|}
\hline $\begin{array}{l}\text { Patient } \\
\text { No./Age, } \\
\text { yr/Sex }\end{array}$ & $\begin{array}{l}\text { Vocal } \\
\text { Fold }\end{array}$ & Muscle & $\begin{array}{l}\text { Insertional } \\
\text { Activity }\end{array}$ & PSA & $\begin{array}{l}\text { Volitional } \\
\text { Activity }\end{array}$ & $\begin{array}{l}\text { Synkinetic } \\
\text { Activity }\end{array}$ \\
\hline \multirow[t]{2}{*}{ 1/52/F } & Right & TA muscle & 2 & 0 & 3 & 3 \\
\hline & & PCA muscle & 1 & 0 & 0 & 0 \\
\hline \multirow[t]{2}{*}{$2 / 46 / F$} & Right & TA muscle & 2 & 0 & 4 & 0 \\
\hline & & PCA muscle & 2 & 0 & 3 & 3 \\
\hline \multirow[t]{2}{*}{$3 / 65 / F$} & Right & TA muscle & 3 & 2 & 0 & 0 \\
\hline & & PCA muscle & 2 & 0 & 1 & 2 \\
\hline \multirow[t]{2}{*}{ 4/39/F } & Right & TA muscle & 2 & 0 & 4 & 3 \\
\hline & & PCA muscle & 2 & 0 & 1 & 0 \\
\hline \multirow[t]{2}{*}{ 5/75/M } & Right & TA muscle & \multicolumn{4}{|c|}{ Data not obtained } \\
\hline & & PCA muscle & \multicolumn{4}{|c|}{ Data not obtained } \\
\hline \multirow[t]{2}{*}{ 6/35/F } & Right & TA muscle & 2 & 1 & 2 & 0 \\
\hline & & PCA muscle & 2 & 0 & 1 & 0 \\
\hline \multirow[t]{2}{*}{$7 / 68 / F$} & Right & TA muscle & 2 & 1 & 2 & 1 \\
\hline & & PCA muscle & \multicolumn{4}{|c|}{ Data not obtained } \\
\hline \multirow[t]{2}{*}{ 8/34/F } & Right & TA muscle & 2 & 1 & 3 & 1 \\
\hline & & PCA muscle & 2 & 0 & 3 & 2 \\
\hline \multirow[t]{2}{*}{ 9/71/F } & Left & TA muscle & 1 & 0 & 1 & 2 \\
\hline & & PCA muscle & 0 & 0 & 2 & 1 \\
\hline \multirow[t]{2}{*}{$10 / 64 / F$} & Right & TA muscle & 2 & 0 & 3 & 0 \\
\hline & & PCA muscle & 2 & 0 & 2 & 2 \\
\hline
\end{tabular}

Insertional activity: 0: none, 1: reduced, 2: normal, 3: increased. PSA: none: 0 , sparse: 1 , clear: 2 , strong: 3 . Volitional activity: none: 0 , single fiber activity: 1 , very reduced: 2 , mildly reduced: 3 , dense: 4 . Synkinetic activity: none: 0 , sparse: 1 , clear: 2 , strong: 3 .

$\mathrm{F}=$ female; $\mathrm{M}=$ male; PCA: posterior cricoarytenoid muscles; $\mathrm{PSA}=$ pathological spontaneous activity; TA = thyroarytenoid muscle . swallowing, and overall satisfaction were evaluated by the Quality of Life Questionnaire of the Lausanne team. ${ }^{23}$

\section{Assessment of Vocal Cord Movement}

Indirect endoscopy. Vocal cord movement recovery was assessed by telescopic laryngoscopy using a $70^{\circ}$ rigid endoscope (Karl Storz, Tuttlingen, Germany) with the aid of topical anesthesia (tetracaine) sprayed into the oropharynx. The videos were analyzed by two laryngologists. The samples were presented in a random order with respect to surgery and blinded with respect to the patient's identity. The capacity for vocal cord adduction was evaluated on each side: none: 0 , minimal: 1 , normal: 2 , able to cross the middle line: 3 .

LEMG. Standard transcutaneous LEMG was performed according to the guidelines of the European Laryngological Society on the side of the larynx in which the adduction movements were more definitely visible. ${ }^{24,25}$ A Nihon Kohden Neuropack X1 EMG system (Nihon Kohden Corp., Tokyo, Japan) with concentric needle electrodes $(50 \mathrm{~mm} \times 26 \mathrm{G}, 37 \mathrm{~mm} \times 26 \mathrm{G}$; Teca elite; Natus Neurology, Pleasanton, CA) were used for the measurements. The data were analyzed with MATLAB R2016a (https://www. mathworks.com; MathWorks, Natick, MA) and Audacity 2.1.3 software (http://audacityteam.org) by a group of three physicians who specialized in laryngeal electrophysiology. ${ }^{26}$ The thyroarytenoid (TA) and the posterior cricoarytenoid muscles (PCA) were successfully examined by LEMG in nine and eight patients, respectively. The following characteristics were evaluated: insertional activity (none: 0 , reduced: 1, normal: 2, increased: 3); pathological spontaneous activity such as fibrillation potentials, myotonic discharges, complex repetitive discharges

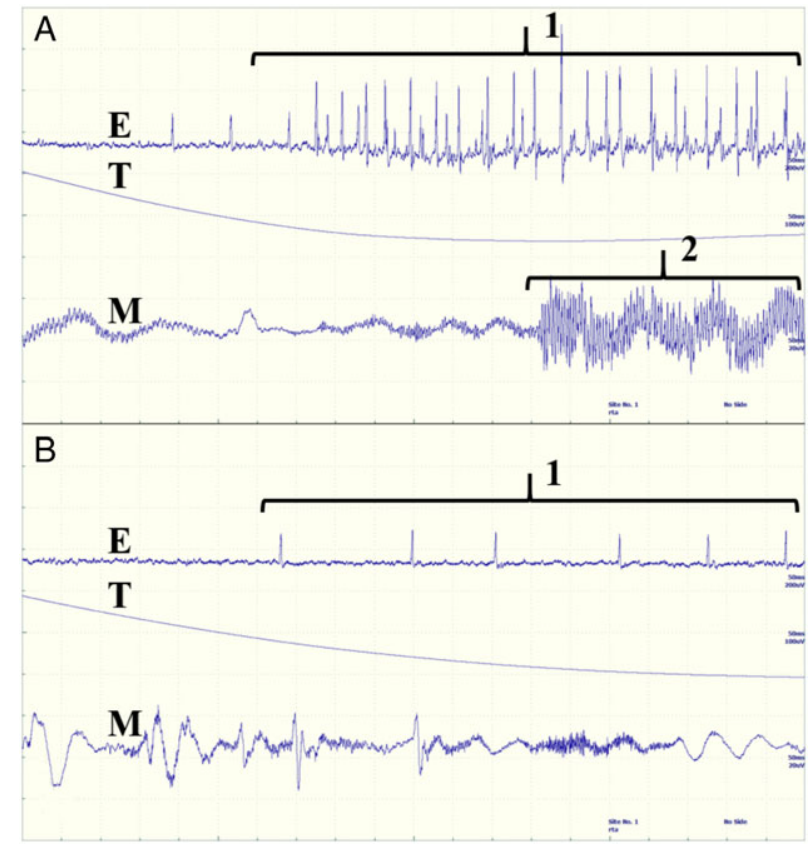

Fig. 2. Laryngeal electromyography of the left thyroarytenoid muscle in a 68-year-old female bilateral vocal cord paralysis patient (patient 4, 12th postoperative month). (A) Very reduced volitional activity (1) of the thyroarytenoid muscle during phonation (2). (B) Sparse synkinetic activity (1) of the thyroarytenoid muscle during inspiration. $\mathrm{E}=$ electromyography; $\mathrm{M}=$ microphone; $\mathrm{T}=$ thermometer. [Color figure can be viewed in the online issue, which is available at www.laryngoscope.com.] [Color figure can be viewed in the online issue, which is available at www.laryngoscope.com.] 
and fasciculations (none: 0 , sparse: 1 , clear: 2 , strong: 3 ); volitional activity (none: 0 , single fiber activity: 1 , very reduced: 2, mildly reduced: 3 , dense interference pattern: 4); synkinetic activity (none: 0 , sparse: 1 , clear: 2 , strong: 3 ).

\section{RESULTS}

\section{Endoscopic Results}

Vocal cord motion recovery is reported in Table I. During the 1-year follow-up, 18 vocal cords showed

A

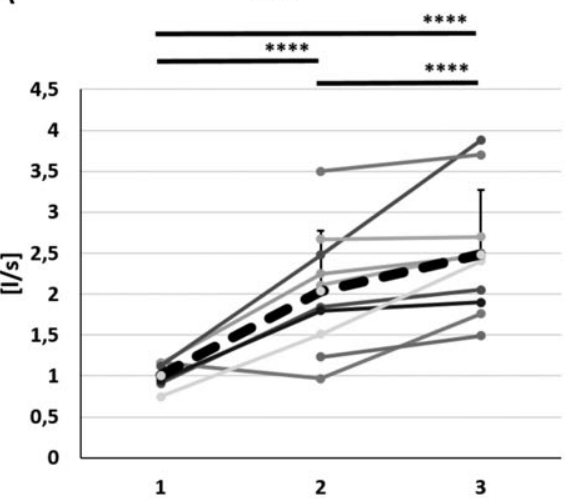

C

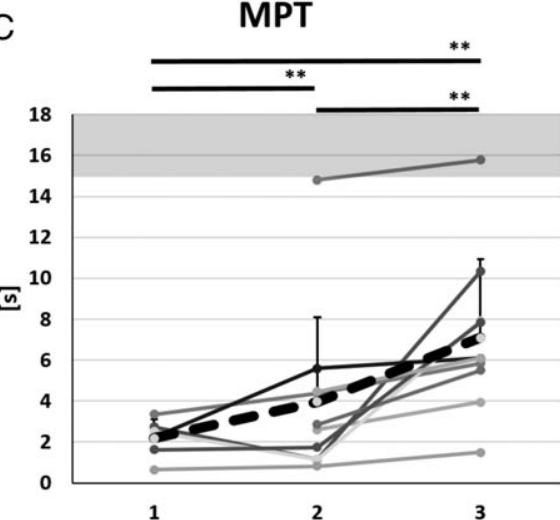

E

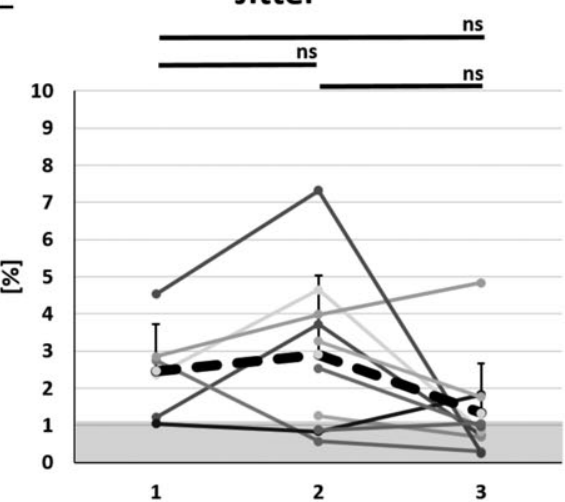

adduction movements. In eight of 10 patients, the nonlateralized vocal cord showed complete or overadduction. In patient 2 and 3 clear, though incomplete adduction was seen in the non-lateralized side. Two patients had more movement at a year in the lateralized side than in the unoperated side (patient 2 and 9 ). In those patients, the lateralized side crossed the midline to contact the other cord. In total, eight of the 10 lateralized cords could visibly adduct at the end of 1 year.
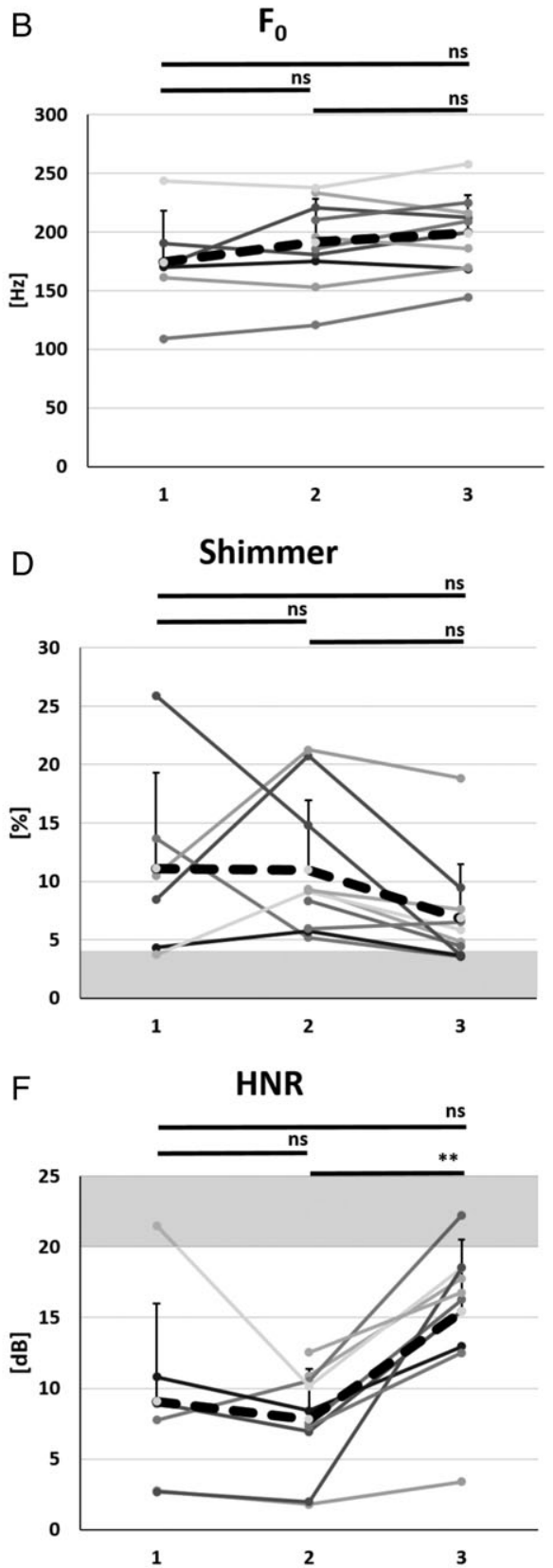

Fig. 3. Objective respiratory and voice results after unilateral endoscopic arytenoid abduction lateropexy in bilateral vocal cord paralysis patients with adduction regeneration. Thin lines represent the changing of the individual values, and the thick-dashed lines demonstrate the average of the values. The gray area represents the normal range of values. 1: preoperative measurement, 2: first postoperative week, 3: first postoperative year. $(* P \leq .05, * * P \leq .01, * * * * P \leq .001)$. (A) Peak inspiratory flow (PIF) (significant improvement). (B) Fundamental frequency (F0) (nonsignificant change). (C) Maximum phonation time (MPT) (significant improvement). (D) Shimmer (nonsignificant improvement). (E) Jitter (nonsignificant improvement). (F) Harmonics-to-noise ratio (HNR) (significant improvement). ns = nonsignificant. 

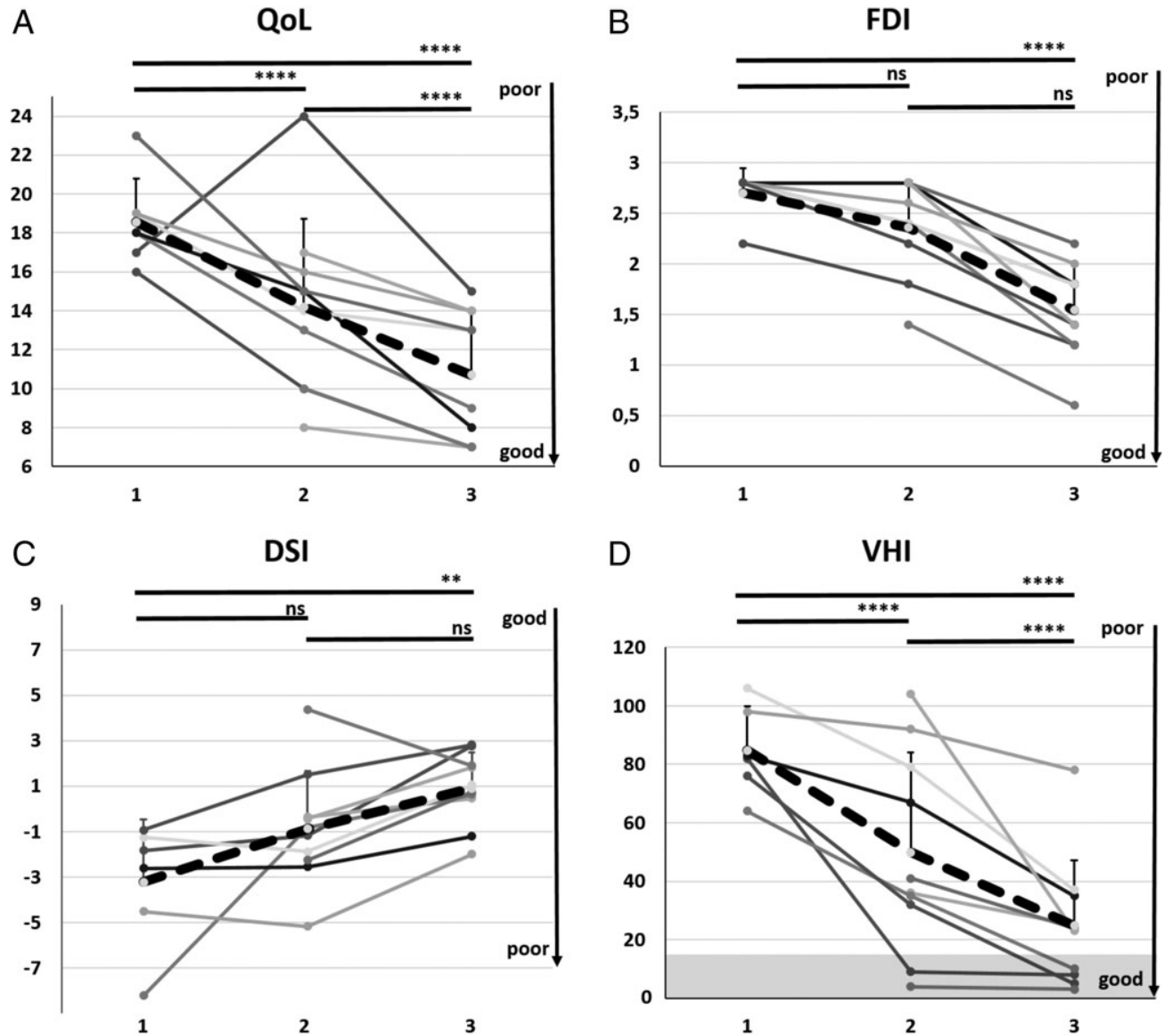

Fig. 4. Results of subjective respiratory and complex voice analysis panel after unilateral endoscopic arytenoid abduction lateropexy in bilateral vocal cord paralysis patients with adduction regeneration. Thin lines represent the changing of the individual values, the thick-dashed lines demonstrate the average of the values. The gray area represents the normal range of values. 1: preoperative measurement, 2: first postoperative week, 3: first postoperative year. (*P $\leq .05$, **P $\leq .01$, ****P $\leq .001$ ). (A) Quality-of-life score (QoL) (significant improvement). (B) Friedrich Dysphonia Index (FDI) (significant improvement). (C) Dysphonia Severity Index (DSI) (significant improvement). (D) Voice Handicap Index (VHI) score (significant improvement). ns = nonsignificant.

\section{Laryngeal Electromyography Results}

The results of LEMG are presented in Table II. Volitional motor unit action potential (MUAP) activity of the TA was observed in eight of nine cases (9/10 cases successfully having LEMG exams) with differing intensities during phonation; however, the interference pattern of the MAUP's was dense in only two cases. In the same muscle, MUAP's indicating synkinesis was detected in five cases (Fig. 2a,b); this means that there was increased electrical activity during inspiration. Nonetheless, the ordinal scale of LEMG detection and degree of movement seen on endoscopy was correlative with only one exception (patient 2).

In seven of eight cases, volitional (appropriate) MUAP activity was detected in the PCA (two were not recorded because they could not tolerate the LEMG exam of the PCA in one case and of any muscle in a second case). A dense interference pattern was not detected in any of them. No visible abduction was seen in any of the larynges of these patients (this was an inclusion criterion). The mean degree of appropriate MUAP activity was 1.6 based on the scale. The same patients' mean MUAP for the TA muscle was $2.5 \pm 1.3$. Synkinetic
MUAP activity of the PCA muscle (increased electrical activity during phonation) was detected in $5 / 8$ cases.

\section{Respiratory Results}

All 10 patients showed objective and immediate airway improvement after unilateral EAAL. The average PIF increased significantly immediately from 1.01 to $2.04 \mathrm{~L} / \mathrm{sec}$ (change $1.03 \mathrm{~L} / \mathrm{sec}, 201.95 \%$ of baseline; $P<.001$ ). This parameter increased later to average of $2.49 \mathrm{~L} / \mathrm{sec}$ (Fig. 3a). The significant improvement of quality-of-life scores also showed the patients' improved satisfaction with their respiratory function (Fig. 4a). The average score improved in the early postoperative period from 18.6 to 14.2 , then further improved to $10.7(P<.001)$.

\section{Voice Results}

The average fundamental frequency of the 10 patients slightly increased in the early and then more in the late postoperative period (109.77\% of baseline and $114.09 \%$ of baseline respectively) (Fig. 3b). The average MPT improvement was 1.78 seconds in the early postoperative period. It 
increased significantly to 7.08 seconds (Fig. 3c) in parallel with the improving vocal cord movements. The value of shimmer showed continuous improvement and decreased to within the physiological range $(\leq 6.84 \%)$ (Fig. 3d)]. Jitter and HNR slightly deteriorated right after surgery, but HNR increased significantly $(P<.01)$ during the regeneration phase compared to the early postoperative period. Jitter showed notable improvement as well, but this did not reach statistical significance (Fig. $3 \mathrm{e}, \mathrm{f}$ ). The complex voice analysis panels verified the improvement of the voice in general. The FDI and the DSI (nonsignificantly) improved immediately after EAAL, but showed further improvement with the regeneration process. Significant improvement (FDI: $P<.001$; DSI: $P<.01$ ) was found between the preoperative and late postoperative values (Fig. 4b,c). The Hungarian VHI demonstrated that patients subjectively also found their voices improved (Fig. 4d). The average score decreased significantly from 84.9 to 49.9 , then to 24.8 (decrease of 59.5 in total; $P<.001$ ).

\section{DISCUSSION}

The human larynx appears designed to default to a rudimentary sphincter function from bilateral RLN injury, with the vocal cords lying in a flaccid paramedian position while there is no airflow. This can allow for some degree of respiration and even phonation, which is commonly possible but tends to be of low volume and very low efficiency. Lower airway protection from aspiration becomes a passive process and often very limited. However, during inspiration, the Bernoulli principle explains the acceleration of the air column between the narrowing of the cords that can, by the generation of a Venturi vacuum, cause a further collapse of the glottic structures because no active abduction can be used to resist this movement. Clinically, this results in inspiratory stridor and necessitates urgent, if not emergency, surgical intervention.

The postinjury equilibrium of adduction and abduction forces from reinnervation clearly changes over time. The complexity of laryngeal innervation (e.g., Galen's anastomosis, the human communicating nerve) may be responsible for the residual innervation and may also explain or at least contribute significantly to reinnervation. ${ }^{27-29}$ However, reinnervation is frequently synkinetic rather than functional, which may be based on the braided mixing of axons that serve antagonistic functions. ${ }^{6}$ Furthermore, the significant numerical superiority of the adductor muscle fibers and axons leads to preferential reinnervation of adduction muscles and adduction during phonation as well as inhalation. ${ }^{8}$ Variations in the position and function of the paralyzed vocal cords could be dependent on the degree and distribution of intrinsic laryngeal muscle reinnervation and synkinesis. Accordingly, BVCP generally does not mean a dead larynx. Quite to the contrary, in most cases, significant volitional adduction can be observed within weeks or months after nerve injury. Conversely, abduction is not known to be as common, occurring in about $30 \%$ of the larger cohort, from which the 10 patients for this report were chosen, whereas about $75 \%$ recovered adduction.

LEMG has been used for decades in the evaluation of vocal cord motion impairment. The technique was reinvented in the recent past and has become an essential tool of the patient selection for laryngeal pacing implantation. ${ }^{24,30}$ Volitional MUAP activity of the TA muscles was detected in 18 of the 20 vocal cords (or nine larynges within the 10 cases who tolerated LEMG) during phonation. Despite the frequency of synkinetic activity detection (in five of nine cases), the phenomenon of preferential reinnervation of the adductor muscles as a functional outcome is strongly supported by our results. The outcome of this hybrid pattern of reinnervation and the resultant vector between the adduction and abduction forces are the basis of an endoscopically obvious volitional adduction motion that enables phonation. The technically more challenging LEMG of the PCA was feasible in only eight of 10 cases. Of the eight, synkinetic activity in the PCA was detected in five of these patients, none of whom were seen to have functional or visible abduction. Therefore, although electrical reinnervation of the PCA (which should cause abduction) could be demonstrated, it was clearly overpowered by the adduction from TA reinnervation. This emphasizes the need for endoscopic assessment to evaluate glottic function regardless of the LEMG results. This also validates the concept that reinnervation is functionally selective even if nervous input is going to both the PCA and adducting muscles like the TA.

In the case of BVCP, the ultimate goal is the rehabilitation of the all three laryngeal functions, respiration, deglutition, and phonation. The conventional static glottis widening techniques, such as arytenoidectomy or transverse cordotomy, do not take advantage of the potential for adduction recovery because they are based on partial or complete resection of the arytenoid cartilage(s) or the vocal cord(s). ${ }^{2}$ In the case of these interventions, dysphagia, aspiration, and hypophonia are common side effects. In addition, the removal of the interarytenoid mucosa, or its postsurgical atrophy, may also increase the risk of aspiration by producing gaps in the protective rim of the laryngeal inlet. Selective reinnervation procedures, or laryngeal pacing, which may restore the physiological mobility of the vocal cord, might be the ideal solutions for this problem. ${ }^{31,32}$ However, nowadays, these surgeries are performed only in a few centers around the globe.

We propose the strong adduction recovery potential found in the larynx following BVCP can be used for the generation of active phonation and good airway protection, in parallel with an adequate airway. This is primarily predicated on the use of minimally invasive glottis widening interventions that do not destroy the voice in the service of airway comfort. The EAAL displaces the entire vocal unit in a physiological manner without resection of phonatory structures and without consequential scarring. The procedure does not damage the interarytenoid region either, which is essential to safe, aspiration-free swallowing. ${ }^{33}$ After the EAAL procedure, the vocal cords become straighter and tenser than after other published endoscopic glottis enlarging interventions. ${ }^{11,12}$ In addition, the EAAL technique produces a relatively small anterior angle compared to other interventions. ${ }^{12}$ This further facilitates better phonatory closure along with improved breathing potential. There are only a few studies in the literature dealing with voice 
quality after glottis-widening surgical interventions. Compared to the commonly used posterior transverse laser cordotomy, EAAL gives better objective and subjective results. Jitter, shimmer, MPT, VHI, FDI, and DSI improved significantly and in parallel with higher peak inspiratory flows. ${ }^{34,35}$ The improvement in objective and subjective voice quality proves that EAAL does not interfere with the potential regeneration process either. Due to the nondestructive manner of the procedure, it also allows active (over) adduction movements on the untreated and even on the lateralized vocal cord if adducting reinnervation occurs. This indicates that if reinnervation occurs, even the lateralized side can return to the service of improved glottic function. The inescapable conclusion is that adduction recovery can overcome the traction of the lateralization suture without the associated damage to the phonatory mechanism known to occur with more traditional approaches such as cordectomy and arytenoidectomy.

\section{CONCLUSION}

EAAL is an easy to perform, safe, and effective solution to BVCP in the properly chosen patients. EAAL allows a simple, quasidynamic option with parallel phonatory and respiratory improvements. This is accomplished by virtue of generating a passively abducted vocal cord without sacrificing the functional integrity of the glottic aperture. This includes allowing for the potential benefits of active adduction recovery in the vocal cords. The long-held premise, that one or two of the primary functions of the larynx (voice quality, swallowing safety, and/or breathing) must be sacrificed to improve the other in BVCP, can now be discarded. We can return a patient to safe, comfortable breathing and a functional voice while avoiding iatrogenic aspiration.

\section{BIBLIOGRAPHY}

1. Belafsky PC. Bilateral vocal fold immobility. Curr Opin Otolaryngol Head Neck Surg 2011;19:415.

2. Sapundzhiev N, Lichtenberger G, Eckel HE, et al. Surgery of adult bilateral vocal fold paralysis in adduction: history and trends. Eur Arch Otorhinolaryngol 2008;265:1501-1514.

3. Grossman M. Contribution to the mutual functional relationship of the muscles of the larynx. Arch Laryngol Rhinol 1906;18:463-471.

4. Woodson GE. Configuration of the glottis in laryngeal paralysis. I: clinical study. Laryngoscope 1993;103:1227-1234.

5. Woodson GE. Configuration of the glottis in laryngeal paralysis. II: animal experiments. Laryngoscope 1993;103:1235-1241.

6. Crumley RL. Laryngeal synkinesis revisited. Ann Otol Rhinol Laryngol 2000;109:365-371.

7. Shindo ML, Herzon GD, Hanson DG, Cain DJ, Sahgal V. Effects of denervation on laryngeal muscles: a canine model. Laryngoscope 1992;102: 663-669.

8. Woodson GE. Spontaneous laryngeal reinnervation after recurrent laryngeal or vagus nerve injury. Ann Otol Rhinol Laryngol 2007;116:57-65.

9. Hirano M, Nozoe I, Shin J, Maeyama T. Electromyography for laryngeal paralysis. In: Hirano M, Kirchner J, Bless D, eds. Neurolaryngology: Recent Advances. Boston, MA: College-Hill; 1987:232-248.

10. Netterville JL, Stone RE, Rainey C, Zealear DL, Ossoff RH. Recurrent laryngeal nerve avulsion for treatment of spastic dysphonia. Ann Otol Rhinol Laryngol 1991;100:100-104.
11. Rovo L, Madani S, Sztano B, et al. A new thread guide instrument for endoscopic arytenoid lateropexy, Laryngoscope 2010;120:2002-2007.

12. Szakacs L, Sztano B, Matievics V, et al. A comparison between transoral glottis-widening techniques for bilateral vocal fold immobility. Laryngoscope 2015;125:2522-2529.

13. Sandhu GS, Nouraei SAR, Rovo L, Marie JP, Mueller AH, Castellanos PF. Bilateral impaired vocal cord mobility. In: Sandhu GS, Nouraei SAR, eds. Laryngeal and Tracheobronchial Stenosis. San Diego, CA: Plural Publishing; 2016:195-227.

14. Rovo L, Jori J, Brzozka M, Czigner J. Airway complication after thyroid surgery: minimally invasive management of bilateral recurrent nerve injury. Laryngoscope 2000;110:140-144.

15. Matievics V, Bach A, Sztano B, et al. Functional outcomes of endoscopic arytenoid abduction lateropexy for unilateral vocal cord paralysis with dyspnea. Eur Arch Otorhinolaryngol 2017;274:3703-3710.

16. Lichtenberger G. Reversible lateralization of the paralyzed vocal cord without tracheostomy. Ann Otol Rhinol Laryngol 2002;111:21-26.

17. Dejonckere PH, Bradley P, Clemente P, et al. A basic protocol for functional assessment of voice pathology, especially for investigating the efficacy of (phonosurgical) treatments and evaluating new assessment techniques. Guideline elaborated by the Committee on Phoniatrics of the European Laryngological Society (ELS). Eur Arch Otorhinolaryngol 2001;258:77-82.

18. Jacobcon $\mathrm{BH}$, Johnson A, Grywalski $\mathrm{C}$, et al. The voice handicap index (VHI) development and validation. Am J Speech Lang Pathol 1997;6: 66-70.

19. Reiter R, Brosch S. Laryngoplasty with hyaluronic acid in patients with unilateral vocal fold paralysis. $J$ Voice $2012 ; 26: 785-791$.

20. Wuyts FL, De Bodt MS, Molenberghs G, et al. The dysphonia severity index: an objective measure of vocal quality based on a multiparameter approach. J Speech Lang Hear Res 2000;43:796-809.

21. Friedrich G. Qualitätssicherung in der Phoniatrie. Vorschlag zur Standardisierung der Klinischen Stimmprüfung. HNO 1996;44:401-416.

22. Cantarella G, Fasano V, Bucchioni E, Domenichini E, Cesana BM. Spirometric and plethysmographic assessment of upper airway obstruction in laryngeal hemiplegia. Ann Otol Rhinol Laryngol 2003;112: 1014-1020.

23. Jaquet Y, Lang F, Pilloud R, Savary M, Monnier P. Partial cricotracheal resection for pediatric subglottic stenosis: long-term outcome in 57 patients. Thorac Cardiovasc Surg 2005;130:726-732.

24. Volk GF, Hagen R, Pototschnig C, et al. Laryngeal electromyography: a proposal for guidelines of the European Laryngological Society. Eur Arch Otorhinolaryngol 2012;269:2227-2245.

25. Bach A, Sztanó B, Kiss JG, et al. The role of laryngeal electromyography in the diagnosis of vocal cord movement disorders [in Hungarian]. Orv Hetil 2018;159:303-311.

26. Sedghamiz H, Santonocito D. Unsupervised detection and classification of motor unit action potentials in intramuscular electromyography signals. The 5th IEEE International Conference on E-Health and BioengineeringEHB At Iasi-Romania. November 19-21, 2015. Available at: https://www. researchgate.net/publication/284362981_Unsupervised_Detection_and_ Classification_of_Motor_Unit_Action_Potentials_in_Intramuscular_ Electromyography_Signals. Accessed March 03, 2016.

27. Sanders I, Mu L. Anatomy of the human internal superior laryngeal nerve. Anat Rec 1998;252:646-656.

28. Henry C, Cazals Y, Gioux M, Didier A, Aran JM, Traissac L. Neuroanatomical study of Galen's anastomosis (nervus laryngeus) in the dog. Acta Anat (Basel) 1988;131:177-181.

29. Wu BL, Sanders I, Mu L, Biller HF. The human communicating nerve. An extension of the external superior laryngeal nerve that innervates the vocal cord. Arch Otolaryngol Head Neck Surg 1994;120:1321-1328.

30. Weddell G, Feinstein B, Pattle RE. The electrical activity of voluntary muscle in man under normal and pathological conditions. Brain 1944; 67: 178-257.

31. Marina MB, Marie JP, Birchall MA. Laryngeal reinnervation for bilateral vocal fold paralysis. Curr Opin Otolaryngol Head Neck Surg 2011;19: 434-438.

32. Mueller AH, Hagen R, Foerster G, Grossmann W, Baumbusch K, Pototschnig C. Laryngeal pacing via an implantable stimulator for the rehabilitation of subjects suffering from bilateral vocal fold paralysis: a prospective first-in-human study. Laryngoscope 2016;126:1810-1816.

33. Crumley RL. Endoscopic laser medial arytenoidectomy for airway management in bilateral laryngeal paralysis. Ann Otol Rhinol Laryngol 1993;102: 81-84.

34. Harnisch W, Brosch S, Schmidt M, Hagen R. Breathing and voice quality after surgical treatment for bilateral vocal cord paralysis. Arch Otolaryngol Head Neck Surg 2008;134:278-284.

35. Misiolek M, Ziora D, Namyslowski G, et al. Long-term results in patients after combined laser total arytenoidectomy with posterior cordectomy for bilateral vocal cord paralysis. Eur Arch Otorhinolaryngol 2007;264: 895-900. 